\title{
„Die Moskauer Sonne scheint überall'6. Die sowjetische Hauptstadt als Raum der Utopie in Das neue Moskau (1938) und Die Schweinepflegerin und der Hirt (1941)
}

\author{
"The Moscow Sun Shines Everywhere". \\ The Soviet Capital as a Utopian Space in New Moscow (1938) \\ and The Swineherd and the Shepherd (1941)
}

\begin{abstract}
Basing on Aleksandr Medvedkin's New Moscow and Ivan Pyryev's The Swineherd and the Shepherd, this case study analyses the way the "new" Moscow was represented as a space of realised utopia in the Soviet socialist realist films of the 1930s and at the beginning of the 1940s. Functioning as a supranational centre of the Soviet "affirmative action empire" (Terry Martin), the cinematographic Moscow casts off all constraints of 'Russianness' in order to become a pan-Soviet model which, both in its architecture and semantics, could epitomize the perfect city and the perfect state. The comparative analysis of both films demonstrates that, although both directors show Moscow through the lens of the so-called "spaces of celebration" (Mikhail Ryklin), "their' Soviet capital does not compensate for the "traumas of the early phases of enforced urbanization", as Ryklin supposed. Rather, it operates as a transformation machine whose impact pertains only to periphery and can be effective once the representatives of this periphery have left Moscow. The complex inclusion and exclusion mechanisms resulting from this logic turn the idealised Soviet capital into a space which only the guests from peripheral regions can perceive as utopian. The ensuing suppression of the inner perspectives on 'utopian' Moscow is interpreted here as a manifestation of the "cinematic unconscious", which accounts for the anxieties of the inhabitants of the capital concerning both Stalinist terror and their own hegemony in a society haunted by the purges.
\end{abstract}

Keywords: utopia, Moscow, cinema, socialist realism, cinematic unconscious, terror

Alexander Chertenko, Justus Liebig University of Giessen, Giessen - Germany, Oleksandr. Chertenko@slavistik.uni-giessen.de, ORCID ID: https://orcid.org/0000-0003-2310-4083

In der sowjetischen kulturellen Produktion der 1930er- und Anfang der 1940er-Jahre - in der Literatur, der bildenden Kunst und vor allem im Kino, dieser „wichtigsten aller Künste“ (Lenin 579), - wird der Moskauer Topos überwiegend 
als Raum der Utopie konstruiert. Als filmische Beispiele hierfür können neben den zwei im Titel genannten Filmen etwa Aleksandr Mačerets Pjotr Vinogradovs Privatleben (Častnaja žizn' Petra Vinogradova, 1934), Grigorij Aleksandrovs Zirkus (Cyrk, 1936), Volga-Volga (1938) und Der helle Weg (Svetlyj put', 1940), Ivan Pyrjevs Das Parteibuch (Partijnyj bilet, 1936), Konstantin Judins Ein Mädchen mit Charakter (Devuška s charakterom, 1939) und Vier Herzen (Serdca četyrech, 1941/1944), Tatjana Lukaševičs Das Findelkind (Podkidyš, 1939) u.a.m. genannt werden. Selbstverständlich blieb Moskau als die ehemalige Hauptstadt des russischen Zarenreichs vor Peter I. und die neue Hauptstadt der Sowjetunion auch vor und nach dieser Zeit nach der Worten Grigorij Revzins eine „Fabrik der Utopien“, „ein Vorraum des «russischen Kosmos»" (Revzin 10; vgl. auch Ljusyj 5-182). Trotzdem entsteht gerade in der Periode des stalinistischen 'Aufbaus' ein besonderes diskursives und visuelles Regime des Moskauer Raums. Dieses geht aus der Verschränkung der projektierten Zukunft und der Gegenwart, die im Zeichen einer „total submission to the official ideology“ (D. Shlapentokh, V. Shlapentokh 73) steht, hervor. Als solches behält es bis in die Perestroika seine Gültigkeit - zuerst als Objekt der Glorifizierung, danach als Ausgangspunkt der Abgrenzung. Janina Urussowa charakterisiert das Kinobild dieses „neuen Moskau“ folgendermaßen:

Die Stadt mit ihren Straßen, ihrem Verkehr und ihren Menschen, die in den Filmen der 20er Jahre noch zu sehen war, verschwand von der Leinwand. Die Realität wurde also durch die von den Künstlern entworfenen und von der Partei befürworteten Zukunftsbilder ersetzt, überblendet und dadurch ausgeblendet. Die Eliminierung der Realität war somit vollbracht. Die Mechanismen des ideologischen Diskurses wandelten den aktuellen Stadttext in ein virtuelles Modell um, an dem von da an die gestalterischen Handlungen vorgenommen wurden. [...] Die Tradition, in der die Hauptstadt Symbolträgerin der politischen Herrschaft war, erwies sich als unüberwindbar (Urussowa 373).

Indem Urussowa die 1920er-Jahre als Vergleichsfolie erwähnt, schließt sie sich an Vladimir Papernyjs Gegenüberstellung der 1920er- und der 1930er-Jahre an, die er in seinem klassischen Buch Kultur ,zwei “ (Kul'tura ,dva") an Beispielen aus der Architektur begründet. In ihm behauptet Papernyj, in den 1930er-Jahren erfolgte in der UdSSR der Übergang von einer „horizontalen“ zu einer „,vertikalen“ Kultur. Die erste, die er „Kultur 1“ nennt, priorisiere „die Wertewelt der Peripherie vor der Wertewelt des Zentrums“. Die zweite (,Kultur 2“) verschiebe vielmehr „die Werte ins Zentrum“, wobei „die Gesellschaft sich kristallisiert und erstarrt" (Papernyj 17). Von dieser These ausgehend, baut Papernyj eine ganze Reihe von Oppositionen auf (Zerfließen vs. Verfestigung, Bewegung vs. Bewegungslosigkeit, gleichmäßig vs. hierarchisch, Improvisation vs. Notation, Realismus vs. Wahrheit usw.), die eine Differenzierung zweier Paradigmen auf verschiedenen Ebenen ermöglichen. Im Bereich der Architektur sieht Papernyj eine 
solche Differenz im Übergang von der Freiheit der Architekten, die „sich selbst überlassen sind und Ideen generieren, die so gut wie nie verwirklicht werden", zur Vereinnahmung der Architektur von Seiten der Macht - ,als eines praktischen Mittels zur Bindung der Bevölkerung an den Raum und als eines räumlichen Ausdrucks des neuen zentripetalen Wertesystems“ (Papernyj 17).

Dieser Übergang bringt natürlich nicht automatisch eine faktische Materialisierung des Projektes mit sich. Als Beispiel sei hier das nicht realisierte Projekt des sog. Palastes der Sowjets (Dvorec Sovetov) genannt, der genauso berühmt ist wie das ebenso nie gebaute Monument für die Dritte Internationale Vladimir Tatlins (1920). Viel wichtiger ist im Kontext der Ästhetik und der Praktiken des sozialistischen Realismus allerdings die Auffassung des Architektonischen als einer verwirklichten Utopie (vgl. z.B. Groys 1995: 143-166; Neutatz 41-44, 53-56; Stites 205-222 u.v.a.) - unabhängig davon, ob das Projekt tatsächlich realisiert wurde, im Entwurf bzw. als Modell blieb oder in seiner fertigen Form von der 'idealen' Vision wesentlich abwich. Man dürfte wohl behaupten, dass die 'Utopie" in diesen Koordinaten eine Form der diskursiven „Selbsthalluzinierung“ darstellt, die das „Wahnsystem“ des Stalinismus (Schlögel 2003: 94) untermauert und diesem zugleich zum Ausdruck verhilft.

Um den Theoretismus der sozrealistischen 'Verwirklichung' zu illustrieren, führt Evgeny Dobrenko ein Zitat aus Vsevolod Lebedevs Glosse Fiktion zwingt uns, Fakten zu lieben (Vymysel zastavljaet nas ljubit' fakty) an, das die Entwicklung des očerk von der Faktografie der 1920er- zur ideologischen Normativität der 1930er-Jahre triftig zusammenfasst:

Ich halte den ruhigen faktografischen Essay (očerk) für unnötig, es bringt keinem etwas bei. Es gibt Naturliebhaber, die die Welt in das Natürliche und das Künstliche klassifizieren und wähnen, dass Wolkenelektrizität die Natur ist und der Dneproges eine Fiktion. Jedoch gibt es tausendmal mehr Natur im Dneproges als in einer Wolke. Dies betrifft auch očerk. [...] Das vom Schriftsteller Geschaffene wird nicht das Plausible (pravdopodobnoe) sein; ins Leben der Leser wird es als eine Wirklichkeit eingehen (zit. nach Dobrenko 2007: 377)1.

Die so aufgefasste utopische Dimension der stalinistischen Architektur der 1930er-Jahre macht einen doppelten architektonisch-filmischen Gestus notwendig. Die Architektur, oder besser gesagt der Bauplan, bildet die materielle Basis, das Gerüst der Utopie - wiederum unabhängig davon, ob es tatsächlich zur Realisierung kam. Wie Karl Schlögel in einer Passage über den Stalinschen General-

1 „Я считаю ненужным спокойный фактографический очерк, он ничему не учит. Есть такие любители природы, которые делят мир на естественное и искусственное, которым кажется, что электричество в туче - это природа, а Днепрогэс - выдумка. Однако в Днепрогэсе этой самой природы в тысячи раз больше, чем в туче. Таков и очерк. [...] Созданное писателем будет не то, что кажется правдоподобным, оно войдет в жизнь читателя как действительность“. 
plan zur Rekonstruktion der Stadt Moskau (1935) zu Recht bemerkt, verwandelt die vom Projekt vorgesehene architektonische Umgestaltung des urbanen Raums diesen bereits zum „Gesamtkunstwerk“, das einen ,integralen Zugriff auf alle Probleme der Stadt"“ voraussetzt:

Es gehörte zur gigantischen Dimension des Projekts, dass es seine eigenen Formen, seine eigene Ästhetik, seine eigene Sprache hervorbrachte, ging es doch um nicht weniger als die Umgestaltung der Welt, die Gestaltung einer neuen Stadt- und Menschenlandschaft aus einem Guss (Schlögel 2008: 65).

Damit das ,aus einem Guss“ gestaltete Idealbild nicht nur entworfen, sondern auch als solches 'gelesen' werden kann, ${ }^{2}$ ist aber ein anderes - visuelles und zugleich erzählendes - Medium notwendig. Dieses macht den spezifischen Mimetismus des sozialistischen Realismus, dessen Referent die „schon von der Regie Stalins und der Partei geformt[e]“ Wirklichkeit (Groys 1988: 62), ja die „,neuesten Richtlinien der Partei“ und der „Wille[] Stalins“ sind (Groys 1988: 59), als ,ein hieroglyphischer Text, den man lesen muss wie eine Ikone, wie einen Leitartikel“" (Groys 1988: 63), erst wahrnehmbar. In der sowjetischen Kunst der 1930er- und 1940er-Jahre wird diese Aufgabe wohl am häufigsten mit kinematografischen Mitteln gelöst (Dobrenko 2008: 4). Denn ausgerechnet die Sprache des Films lässt jene fingierte Visualität des Sozrealismus in großem Stil erscheinen, in deren Rahmen „die visuelle Prozedur die von ihr hervorgebrachten verbalen Operationen nur kaschiert“ und deren Rezipient sich in der „Kontextualisierung und Interpretation des Gesehenen“ „übt“ (Dobrenko 2007: 383). Mit Janina Urussowa darf man wohl behaupten, dass, während die Architekten ,eine Art Universaliengerüst der Ideologie“ (Jakovleva 30) auf die Stadt legen, die Filmemacher „dieses Gerüst der Universalien filmen und [...] aus den Aufnahmen eine Idealstadt zusammen[schneiden]“ (Urussowa 371), die sich ohne „sehende Sprache“ des Films der Lektüre entzieht, ja kaum existiert.

$$
* * *
$$

In den 1930er- und Anfang 1940er-Jahren erweist sich die sowjetische Hauptstadt als die einzige sowjetische Stadt, die mit Hilfe dieses zweistufigen Prozesses als verwirklichte Utopie, als Gesamtkunstwerk in vollem Ausmaß konstruiert und präsentiert werden kann. Neben einer grundsätzlichen Irreproduzierbarkeit der Utopien bedingte vor allem der Zentralismus der stalinistischen Kulturpoli-

2 Auf 'Lesbarkeit' als ein konstitutives Element des Moskauer Generalplans von 1935 weist auch Oksana Bulgakowa hin: „Die Stadt verwandelte sich allmählich in ein codiertes Buch, das gelesen werden musste, doch die Schrift erschloss sich nicht jedem. Der Plan sollte der Morphologie der Stadt zur Sinnträchtigkeit verhelfen“ (Bulgakowa 261). 
tik die Exklusivität des Moskauer Topos. Neben der totalitären Dimension hat dieser Zentralismus auch einen spezifischen imperialen Aspekt, der sich in den Koordinaten von Terry Martins ,affirmative action empire“ wohl am besten beschreiben lässt. Genauso wie das russische Volk laut Martin auf seine „,national interests" verzichtet, um ,the Soviet Union's state-bearing people“ zu bleiben und „to further the cohesion of the multinational state" (Martin 20), verzichtet das gefilmte Moskau der 1930er-Jahre als eine „verwirklichte Utopie“ auf ihren nationalen (russischen) Status. Hierdurch wird es umso effektiver zu einem gesamtsowjetischen Modell, das per definitionem einmalig ist. Als solches fokussiert es die ideellen Dimensionen des damaligen Kultur- und Staatsaufbaus in sich und verkörpert architektonisch wie semantisch das Muster der perfekten Stadt, im Endeffekt auch des perfekten Staates.

Die prinzipielle Novität eines solchen „,neuen Moskau“ zeigt einerseits dessen Vergleich mit sowjetischen urbanistischen Filmutopien der 1920er-, andererseits mit den filmischen Darstellungen der Idealstädte der 1930er-Jahre jenseits des Sadovoe kol'co. Im ersten Fall bietet sich der berühmte Film Dziga Vertovs Der Mann mit der Kamera (Čelovek s kinoapparatom, 1929), in dem Moskau neben Kyiv und Odesa als Inbegriff der Fortschrittsleistungen der Moderne auftritt, als Beispiel an. Ganz im Sinne der dezentralen Logik einer „Kultur 1“ werden in ihm drei Großstädte mit Hilfe raffinierter Montagetechniken gleichermaßen entrealisiert und auf basale Elemente der modernen Urbanität zurückgeführt. Im Rahmen einer symphonieartigen Komposition - man denke hier an den Titel des wegweisenden Films Walter Ruttmanns (Die Sinfonie der Großstadt, 1927) - werden diese Elemente zu Bausteinen einer ,genuin internationalen, absoluten Kinosprache“63 und zugleich zu Facetten einer zukunftsträchtigen Welt der Technik. Hier geht es weniger um das Muster, das die Zukünftigkeit der sozialistischen Gegenwart sichtbar und lesbar macht, sondern vielmehr um eine mosaikartige Darstellung der Städte (nicht: der Stadt) und der Technik der Gegenwart, die als zukunftsweisend verstanden werden. Im zweiten Fall kann auf die Filme wie Chabarda (1931; Regie Michail Čiaureli, Drehbuch Sergej Tretjakov) oder Aerograd (1935; Regie und Drehbuch Oleksandr Dovženko) hingewiesen werden. Im Unterschied zu Vertovs Der Mann mit der Kamera - und in Übereinstimmung mit dem sozrealistischen Zentralismusprinzip - werden die peripheren urbanen Utopien, die in Tiflis/Tbilisi (Chabarda) oder an der Pazifikküste (Aerograd) liegen, in beiden Filmen als (noch) nicht realisierte Projekte, bestenfalls als work in progress dargestellt. Hierdurch deuten auch sie indirekt die unsichtbare Präsenz des Moskauer Zentrums an, das andere urbane Utopien nur dann zulässt, wenn diese die „state-bearing function" Moskaus anerkennen und die Hauptstadt als Musterprojekt bestätigen.

\footnotetext{
3 „Подлинно международный абсолютный язык кино“.
} 
Als historische Parallele zu dieser Lesart kann die Geschichte des Wiederaufbaus von Minsk nach dem Ende des Zweiten Weltkriegs dienen. Wie Thomas Bohn zeigt, haben die Autoren des Generalplans von 1946 schon früh den Kurs der Utopie einer „original sozialistischen Stadt“ eingeschlagen. Dementsprechend gewann dort ein Projekt Oberhand, das sich als Kopie des Moskauer Generalplans von 1935 verstand und so gut wie keine lokalen Besonderheiten oder stilistischen Traditionen berücksichtigte (Bohn 81-88).

Im Kontext der Privilegierung Moskaus als einer real existierenden Utopie, die sich in der sowjetischen Kinokunst der 1930er- und Anfang 1940er-Jahre abzeichnet und den politischen Prozessen jener Zeit einen idealtypischen Ausdruck verleiht, stellt sich die Frage nach den neuen Verhältnissen zwischen Zentrum und Peripherie, Stadt und Land, die hiermit einhergehen und die kinematografischen Darstellungen der sowjetischen Hauptstadt strukturieren. Aus welcher Perspektive wird das Zentrum des totalitären „affirmative action empire“, das als Gesamtkunstwerk konzipiert ist, in den Filmen der 1930er- und Anfang 1940erJahre (re-)konstruiert? Wie werden die Verhältnisse zwischen diesem Zentrum und den peripheren, ggf. nicht-urbanen Landschaften und deren Bewohner aufgebaut? Welche Prozeduren und Effekte der In- und Exklusion werden hierdurch gefördert? Dies versuche ich im Weiteren, durch vergleichende Analyse zweier exemplarischer Filme jener Jahre zu verdeutlichen: Aleksandr Medvedkins Das neue Moskau (Novaja Moskva, 1938) und Ivan Pyrjevs Die Schweinepflegerin und der Hirt (Svinarka i pastuch, 1941; im Folgenden als Die Schweinepflegerin angesprochen $)^{4}$.

$$
* * *
$$

Die zwei sehr unterschiedlich konzipierten und künstlerisch umgesetzten Filme repräsentieren hier zwei Pole der kinematografischen Moskau-Darstellungen in der stalinistischen Vorkriegszeit. Der in mehrfacher Hinsicht experimentelle Film Medvedkins, eines Regisseurs, der zwischen Avantgarde und sozialistischem Realismus oszilliert, stellt einen Versuch dar, den Generalplan zur Rekonstruktion Moskaus in betont phantastische Bilder und zugleich in die Gattungssprache der Liebeskomödie zu übersetzen. Der Film Pyrjevs gehört, wie die meisten Produktionen des Regisseurs, zum Genre der musikalischen Komödie und thematisiert - ebenso wie die meisten seiner Filme - die Verhältnisse ,entlang der Achse «Peripherie - Zentrum»" (Dobrenko 2007: 532), in denen Moskau die Rolle einer normbildenden Instanz zugewiesen ist. In Das neue Moskau, wie schon der Titel verrät, spielt sich die Handlung überwiegend in Moskau und Umgebung

${ }^{4}$ Im deutschen Filmverleih als Sie trafen sich in Moskau bekannt. Beide Filme werden - allerdings nur beiläufig - in der Medvedkin-Monografie von Emma Widdis verglichen (Widdis 106). 
ab und wird nur am Anfang und Ende in die entlegenen Regionen verlagert. In Die Schweinepflegerin, wie dies ebenfalls aus dem Titel hervorgeht, dominiert die ländliche Provinz. Moskau wird in Pyrjevs Film lediglich in zwei Episoden gezeigt und beschränkt sich auch dort auf die berühmte Ausstellung der Errungenschaften der Volkswirtschaft der UdSSR (Vsesojuznaja sel'skochozjajstvennaja vystavka, kurz VSChV). Unterschiedlich gestaltete sich schließlich auch die Rezeptionsgeschichte beider Filme. Medvedkins Film wurde nicht herausgebracht - wie Janina Urussowa vermutet, vor allem weil die vom Regisseur inszenierten Bilder der Moskauer Utopie „ideologisch zu kurz gekommen“ waren und die Klassenzugehörigkeit der Protagonistin Zoja Novikova (Nina Alisova) als kleinbürgerlich empfunden wurde (Urussowa 346-347). Pyrjevs Film, der erst nach dem Ausbruch des „Großen Vaterländischen Kriegs“ abgeschlossen wurde, erfreute sich im Gegenteil bei dem damaligen Publikum großer Beliebtheit und gewann im Jahr 1942 sogar den Stalinpreis.

Trotz dieser Unterschiede im Genre, dem Umgang mit Raum und der Rezeptionsgeschichte haben beide Filme bezüglich des Moskau-Narrativs wichtige strukturelle Elemente gemeinsam, die auch für die sozrealistischen Darstellungs- und Denkweisen der 1930er-Jahre charakteristisch sind. Am offensichtlichsten sind die Parallelen in der territorialen und kulturellen Zugehörigkeit der handelnden Personen. So kommen die meisten Figuren in Das nеue Moskau und alle Figuren in Die Schweinepflegerin, die mit Moskau auf verschiedene Weise interagieren, aus der Peripherie bzw. identifizieren sich mit ihr. In Medvedkins Film lebt der Ingenieur Aljoša Konopljannikov (Daniil Sagal), selbst ein Moskauer, nicht in der Hauptstadt. Zusammen mit anderen Ingenieuren, die allesamt ebenso aus Moskau stammen, beschäftigt er sich mit der Entsumpfung der Taiga, 3000 km von Moskau entfernt. Dort entwirft er das Projekt einer idealen (fiktiven) Kleinstadt Vypino, welches die utopische Dimension des Moskauer Generalplans en miniature wiedergibt. Aljošas Leben weit von der sowjetischen Hauptstadt wird im Film konsequent als die freie Wahl eines Menschen interpretiert, der dort bleibt, wo „,ihn unsere blühende Heimat am meisten braucht" ${ }^{\text {“5 }}$ Dies führt zum Verlust der Identifikation mit Moskau. Am Anfang des Films halten die Ingenieure von Vypino es noch für notwendig, mit Aljoša, der das „lebendige Modell Moskaus"“6 in die Hauptstadt transportiert - möglicherweise ein provinzielles Pendant zum realen elektrischen Modell, das u.a. von Lion Feuchtwanger beschrieben wurde (Feuchtwanger 35-36) -, die alte Babuschka (Marija Bljumental'-Tamarina) zu entsenden, damit der Protagonist garantiert zurückkommt. Am Ende kommt für Aljoša etwas anderes als sein frohes Schaffen an der Peripherie nicht mehr in

\footnotetext{
5, „[...] где он больше всего нужен нашей цветущей родине“.

6 „Живая модель Москвы“.
} 
Frage: „Wir lieben den Bau nicht weniger als Moskau“7. In Die Schweinepflegerin geraten die Moskauer gar nicht in den Fokus. Beide Protagonisten und die durch sie vertretenen Figurengruppen stehen für zwei weit voneinander entfernte Peripherien. Der Schafzüchter Musaib Gataev (Vladimir Zel'din), der sich als Spitzenarbeiter einen Namen machte, lebt in einem winzigen Aul in Dagestan und repräsentiert somit den orientalen, rückständigen und edlen Kaukasus. Die Schweinezüchterin Glaša Novikova (Marina Ladynina) aus einem Dorf irgendwo in den „nördlichen Wäldern“ (severnye lesa), im Gebiet Wologda, personifiziert eine nicht minder rückständige russische Provinz. Obwohl Glaša und Musaib, genauso wie Aljoša, die Anziehungskraft Moskaus deutlich spüren, können und wollen sie ihre regionale Identität keineswegs abschütteln: „Moskau ist doch riesengroß. Moskau ist doch ein fremder Ort. Und ich bin ein kleines Mädchen aus den nördlichen Wäldern“8.

Eine solche Selbstverortung der Figuren verwandelt das Moskauer Sujet in einen Reisebericht. Auf dem Weg von der Peripherie ins utopische Zentrum sehen sich die dramatis personae mit zahlreichen Hindernissen konfrontiert, die ihre Biografien prägen und den schwierigen Kreuzweg zur , hellen Zukunft' symbolisieren'. So verpasst Aljoša in Medvedkins Film, indem er der Studentin der Moskauer Agrarakademie Olja (Marija Barabanova) hilft, ihr Ferkel zu fangen, seinen Zug nach Moskau und bringt so den Einsatz des „lebendigen Modells“ in Gefahr. Zugleich wird dieser Vorfall zum Startpunkt seiner Liebesgeschichte mit der Moskauerin Zoja. In Pyrjevs Musical erschwert die Trägheit der Provinz weniger die Reise an sich, als deren Wiederholung in veränderten Umständen. Nachdem beide Hauptfiguren mühelos den Weg von der Peripherie ins Zentrum und zurück bewältigen, müssen sie den Widerstand ihrer Umwelt, konkret den des Pferdepflegers Kuz'ma Petrov (Nikolaj Krjučkov) niederkämpfen, der Glaša umwirbt und ihren Briefwechsel mit Musaib unterbindet. Erst nach überstandenem Kampf können sich beide wieder treffen - wenn nicht in Moskau, so doch sozusagen unter Moskauer Schirmherrschaft.

Als Projektionsfläche für die Verhältnisse zwischen Zentrum und Peripherie(n) und zugleich als Medium, das die Wirkungskraft des Zentrums in die Regionen überträgt, dienen in beiden Filmen - ganz im Sinne der sozrealistischen „neuen

\footnotetext{
7 „Стройку мы любим не меньше, чем любим Москву“.

8 „Москва-то она чай огромная. Москва-то она незнакомая. А я девчонка маленька из северных лесов“.

${ }^{9}$ Dass dies auch wörtlich zu verstehen ist, zeigt die burleske Kreuzigungsszene in „Das neue Moskau", in der die 'veraltete' Kunst in Gestalt des Malers Fedja einen symbolischen Tod erlebt (vgl. Drubek-Meyer 248).
} 
Romantik“ (Groys 1988: 66) ${ }^{10}$ - die Liebesgeschichten der Protagonisten. Diese Geschichten werden als eine Begegnung und ein Ineinandergreifen verschiedener kultureller Identitäten erzählt: die der kaukasischen und der russischen in Die Schweinepflegerin; die der metropolitanen (der Maler Fedja Utin (Pavel Suchanov) und Zoja) und der randständigen (Aljoša und Olja) in Das neue Moskau. So macht Aljoša dem Moskauer Fedja dessen Freundin Zoja abspenstig und überzeugt sie, allerdings erst nach mehreren Anläufen, zusammen mit ihm in die Taiga zu übersiedeln. Fedja bleibt anschließend nichts anderes übrig, als sich mit der offenbar vom Lande stammenden Olja zu trösten. Ähnlich kündigt Glaša in Die Schweinepflegerin im letzten Moment ihre monokulturelle Hochzeit mit Kuz'ma und entscheidet sich endgültig für den Dagestaner Musaib.

In diesen Geschichten des Kulturkontakts, die in den matrimoniellen Koordinaten verortet sind und deutlich an das Ideologem der „Völkerfreundschaft“ bzw. der Annäherung von Stadt und Land anknüpfen, ist Moskau mehr als eine Kulisse. Vielmehr wird es buchstäblich zum Bestandteil der Liebesbeziehung. So werden Aljoša und Zoja in Das neue Moskau gerade während der Vorstellung des „lebendigen Modells“"zum ersten Mal als Paar gezeigt: Aljoša zeigt dabei die Bilder und Zoja liest den Begleittext. Und in Die Schweinepflegerin werden im Lied „Es fühlt sich gut an in den Weiten Moskaus..." („Chorošo na moskovskom prostore...") Augen und Worte des (der) Geliebten in einem Atemzug mit den Elementen der Moskauer Geografie genannt:

Ich werde deine strahlenden Augen

und deine einfachen, zärtlichen Worte nie vergessen.

Ich werde auch die schönen

Plätze, Gassen und Brücken in Moskau nie vergessen ${ }^{11}$.

Dass die 'Messages' beider Filme, so unterschiedlich diese sind, eine ähnliche Tendenz ausweisen, zeigen auch einige, manchmal recht eindeutige, Überschneidungen in Details. Ein solches ist etwa das ironisch gefärbte Bild des Schweins (oder des Ferkels). Nicht nur in Die Schweinepflegerin, wo Schweine berechtigterweise eine zentrale Rolle spielen, sondern auch in Das nеие Moskau erscheinen immer wieder Schweine auf der Leinwand. Hier wie dort werden sie als Objekte der Zucht, der Demonstration oder als Versuchstiere zur Schau gestellt. In Das neue Moskau geraten sie außerdem in die prächtigen Räume der Moskauer Metro und bestätigen dort in auffallender Weise die (ursprüngliche) Inkompatibilität

${ }^{10}$ Eine ähnliche Tendenz lässt sich auch im deutschen Kino der 1930er- und ersten Hälfte der 1940er-Jahre erkennen (Brockmann 167-179).

11 „Не забыть мне очей твоих ясных, / и простых твоих ласковых слов. / Не забыть мне московских прекрасных / площадей, переулков, мостов“”. 
ihrer Besitzer mit der sowjetischen Weltstadt - aber auch die Dörflichkeit des heutigen Moskaus im Vergleich zum Moskau von morgen (Urussowa 298-299). Zugleich wird das Schwein zum lebendigen Ausdruck für die Produktivität der provinziellen Interventionen in die ,verwirklichte Utopie“. In Die Schweinepflegerin steigert Glaša durch Einsatz der modernsten Methoden der Schweinezucht, die auf der VSChV präsentiert wurden, die Fruchtbarkeit ihres Schweinebestandes fast ins Unglaubliche. Dieser Erfolg materialisiert die angestrebte Symbiose zwischen der Zeugungskraft des idealen Moskau und dem empfangenden Schoß der Peripherie. In Das neue Moskau bringt die Verfolgung des vor Olja fliehenden Ferkels erst Aljoša und Zoja und dann auch Olja und den von Zoja verlassenen Fedja zusammen. So wird die Fruchtbarkeit des Schweins, die in Die Schweinepflegerin auch im Wortsinn zu verstehen ist, symbolisch auf die zwischenmenschlichen Beziehungen übertragen. Diese Übertragung manifestiert sich u.a. in einer nicht zu übersehenden Ähnlichkeit zwischen den Darstellungen hauptstädtischer Menschenmassen und den Bildern der um die Muttersau wimmelnden Ferkel oder auch der Schafherde in den Bergen von Dagestan.

$$
* * *
$$

Aus diesen gemeinsamen Bauteilen entstehen in beiden Filmen zwei semantisch recht nah stehende Bilder des utopischen Moskaus, denen komplexe Einund Ausschlussprozeduren zugrunde liegen. Das kinematografische Idealbild steht dabei in deutlichem Gegensatz etwa zur Utopie von Thomas Morus, die auf einer Insel situiert und durch das Meer und zahlreiche unsichtbare Hindernisse vom Land getrennt ist, ${ }^{12}$ oder zu Tommaso Campanellas Die Sonnenstadt, die von einer mehrstufigen uneinnehmbaren Mauer umgeben ist (Chertenko 130-132). Ganz anders basiert das Moskaubild von Medvedkin und Pyrjev nicht auf der Abgrenzung, sondern vielmehr auf einer Einbindung der peripheren Außenwelt in den metropolitanen Raum. Eine Folge hiervon ist die Hybridisierung von verschiedenen Klassen und Ethnien im egalitären 'Schmelztiegel' der sowjetischen Hauptstadt. Erst kraft seines ethnonationalen und kulturellen Synkretismus wird Moskau zum Zentrum des ,affirmative action empire“ und erhebt sich als solches über die provinziellen Einschränkungen nationaler oder lokaler Natur. Man darf behaupten, dass das filmische Moskau der 1930er- und Anfang 1940er-Jahre in diesem Punkt anderen Weltstädten ähnelte, vor allem den US-amerikanischen Metropolen, die damals als Höhepunkt der Moderne galten - eine Tatsache, die etwa der US-amerikanische Architekt Frank Lloyd Wright scharf kritisierte (Papernyj 24)

${ }^{12}$ In diesem Punkt möchte ich mich von dem leitmotivischen Vergleich des 'utopischen' Moskau mit der Utopie Thomas Morus abgrenzen, den u.a. Anne Hartmann im Anschluss an die Moskau-Reportage von Karl Schmückle stark macht (Hartmann 103-104, 111-113). 
und die europäischen Intellektuellen wie Lion Feuchtwanger (Urussowa 296) oder Joseph Roth (Voloshchuk 143-144) ohne Sympathie kommentierten.

Zwei Einschränkungen sind dabei zu berücksichtigen. Zum Ersten finden nur die besten Vertreter der jeweiligen Schichten und beruflichen Milieus in die ideale Stadt hinein - seien es Ingenieure, wie in Das neue Moskau, oder Kolchosbauern, wie in Die Schweinepflegerin ${ }^{13}$. In diesem Sinne fungieren die unsichtbaren und scheinbar porösen Mauern um die Moskauer Utopie in traditioneller Manier als Sieb, das alle durchschnittlichen Bewohner der Peripherie herausfiltriert. Als Symbol dieser 'Filtration' ist der handlungsbildende Topos der Ausstellung zu verstehen, zu der nur qualitativ hochwertige Objekte zugelassen werden - wie das „lebendige Modell“", das Zuchtvieh oder die sozialistischen Menschen. Zum Zweiten funktioniert der Moskauer Raum selbst im Hinblick auf die Ankömmlinge als ein „Nicht-Ort“ (Auge). Die angereisten oder zugezogenen Kleinstädter und Dorfbewohner werden durch dessen breite Straßen, die in aller Regel in Totalen dargestellt werden, wie Vieh en masse hindurchgespült - oder durch die etwas engeren, jedoch ebenso vorbildlichen Arterien der VSChV - und nach der vollzogenen ,Umerziehung' zu sozialistischen Menschen wieder herausgedrängt.

Die dem utopischen Moskau innewohnende Hybridisierungstendenz sowie dessen Semantik des Transitorischen drücken sich in beiden Filmen in der Privilegierung der sog. „Räume des Jubels“ aus - der VSChV in Die Schweinepflegerin; der Moskauer Metro, der namenslosen Ausstellung und des ihr vorangehenden Karnevals in Das neue Moskau. Als „Räume des Jubels“ bezeichnet Michail Ryklin jene architektonischen Strukturen der Stalin-Zeit, die als „Paläste[] ohne Palastherrn (also Volkspaläste[]) und Tempel[] ohne Gott“ dastehen, eine „Unterwerfung unter das Kollektiv und Verachtung gegenüber dem Individuum“ darstellen und mit dem „Pathos des «Neuen Lebens» und von oben verordnetem Jubel“ errichtet werden (Ryklin 147). Diese „Tempel-Paläste“ üben laut Ryklin eine kompensatorische Funktion aus. Denn in den Augen der Menschen, die ,in der Hauptsache [...] gestern noch Bauern gewesen“ und ,in ihrem Unbewussten von Archetypen geprägt [sind], die von ihren früheren Unterdrückern entlehnt waren", kompensieren diese paradoxen, im Alltag etwas deplatziert wirkenden Monumentalgebäude „das Trauma der Frühphase der Zwangsurbanisierung, die mit einem horrenden Mangel an privatem Wohnraum und der Dominanz sogenannter Kommunalwohnungen in der Sowjetunion zusammenhing“(Ryklin 143). Indem den „Massen der Stalin-Zeit“ ein kompensatorisches Surrogat angeboten wird, feiert die Ideologie „mit Hilfe von Gewalt den Sieg über den Alltag“ (Ryklin

${ }^{13}$ Emma Widdis betrachtet die Verwandlung Moskaus zu einem ,reward for spectacular labour achievement" als ein typisches Merkmal des filmischen Moskau-Bildes der 1930er- und Anfang 1940er-Jahre (Widdis 97). 
144). So erleben die von der Hauptstadt angelockten „Bauern von gestern“ die verwandelnde Wirkung der verwirklichten Utopie, als die das „neue Moskau“ der 1930er-Jahre angesehen wird.

$* * *$

Dieses theoretische Modell wird allerdings in beiden Filmen um eine wichtige Komponente erweitert. Denn die „Räume des Jubels“, wie Ryklin sie versteht, oktroyieren den ehemaligen Bauern (und heutigen Arbeitern), die sie im Akt der kollektiven Katharsis erleben, kein eindeutiges Verhaltensmodell auf. Nachdem der verklärte sozialistische Mensch den Transitraum etwa der VSChV verlässt, darf er relativ frei davongehen - gesetzt den Fall, er ist grundsätzlich bereit, sich dort einzusetzen, wo die Partei dies für nötig erachtet. Sowohl in Das neue Moskau als auch in Die Schweinepflegerin wird diese Freiheit aber wesentlich eingeschränkt. Hier darf der moskauisierte Hauptstadtgast nicht in beliebige Richtung ziehen, sondern er wird dorthin herausgespült, wo er hergekommen ist: in die Provinz. Es ist in diesem Sinne wohl kein Zufall, dass die Fahrt von Moskau in die Peripherie, anders als von der Peripherie nach Moskau, in beiden Filmen reibungslos verläuft; mehr noch: Sie wird mit der Semantik der Beschleunigung versehen, die im Vergleich mit den Hindernissen auf dem Weg nach Moskau besonders klar hervortritt. So pilgert Zoja in Das neue Moskau, nachdem ihr endlich der Sinn für ihr familiäres (mit Aljoša) und 'ziviles' (auf der Baustelle in Vypino) Glück aufgeht, nicht einfach in die Taiga. Stattdessen fliegt sie mit dem Flugzeug, diesem Wunderwerk der Moderne, das dem provinzialisierten Aljoša als Möglichkeit gar nicht einfällt, dort hin und trifft früher als ihr künftiger Mann ein. In Die Schweinepflegerin reitet der dagestanische Hirt in seiner Burka, in der man ihn vor einer Sekunde noch in Moskau gesehen hat, gleich im nächsten Bild stürmisch in die „nördlichen Wälder" hinein, um dort die Gerechtigkeit wiederherzustellen. Hierbei gewinnt er kraft der Montagetechnik eine Geschwindigkeit, die sein antiquiertes Verkehrsmittel nur durch einen Impuls aus Moskau erreichen kann.

Gerade mit dieser zentrifugalen Bewegung wird in beiden Filmen - in Abweichung von Ryklins Modell - die Hauptwirkung der Moskauer Utopie der 1930erJahre assoziiert. Dementsprechend bewähren sich die in Moskau erlernten Produktions- und Lebensweisen in der Praxis nur jener Figuren und Paare, die im Krähwinkel der tiefsten Provinz wohnen. In Die Schweinepflegerin können z.B. die in Moskau entworfenen und auf der VSChV zur Schau gestellten Regeln der Wissenschaft nur fern von der sowjetischen Hauptstadt, im Alltag des russischen Dorfes oder des kaukasischen Auls, zur vollen Entfaltung kommen. Nach der gleichen Logik bleibt die Liebesgeschichte von Fedja und Olja, die Moskau nicht verlassen wollen, in den ersten Ansätzen stecken. Die Liebesaffäre von Aljoša und Zoja führt im Gegenteil zu einer tiefgreifenden Veränderung nicht nur ihres Pri- 
vatlebens, sondern auch des Baus in Vypino, dessen rasche Fortschritte erst nach dem Eintreffen des Pärchens in der Taiga sichtbar werden.

Noch deutlicher und vielschichtiger zeigt sich der Einfluss dieser zentrifugalen Logik in dem bereits erwähnten „lebendigen Modell“. Als Produkt nicht des Zentrums, sondern des sibirischen Niemandslands ist es ein untrügliches Zeichen für die Teilhabe der Peripherie am Ausbau Moskaus zur Utopie. Dessen avantgardistisch anmutende Bilder der Zukunft, die an die architektonische Kulisse in Fritz Langs Metropolis (1927) erinnern, lassen sich - gerade wegen ihrer peripheren Herkunft - als Andeutung auf die Entkanonisierung der Avantgarde (und der avantgardistischen Utopie) in der Zeit des Sozrealismus interpretieren. Obwohl das „lebendige Modell" nur in der Hauptstadt Sinn hat, macht sich dessen Wirkung - genauso wie die Wirkung der VSChV in Die Schweinepflegerin - erst in der Peripherie bemerkbar, dort, wo es herkam und wohin es einen veränderten Alltag und neue Beziehungen bringt. Nur in dieser Funktion - als Ahnung der 'moskauisierten' Peripherie und nicht eines Moskau von morgen - wird das Moskau-Modell im Film Medvedkins regelmäßig zum Anlass, das Verhalten der handelnden Figuren zu überdenken und neu zu bewerten. Dies passiert etwa dann, wenn Aljoša die Moskauerin Zoja als „keine Patriotin“ (ne patriotka) bezeichnet, da sie sich von der Vorstellung der Moskau-Reproduktion in der Taiga nicht ohne weiteres faszinieren lässt und sich stattdessen an den Bequemlichkeiten des real existierenden Moskau wie Piroggen mit Sauerkraut, Gas- und Zentralwasserversorgung festhält. Dasselbe Prinzip wird im Lied des Protagonisten als Vergleich Moskaus mit der Sonne formuliert, die das ganze sowjetische Territorium (u.a. auch Vypino) erwärmt:

Die Moskauer Sonne scheint überall,
überall schickt sie ihre Strahlen aus $[\ldots]$
Moskau beflügelt jeden von uns
und bringt uns das Arbeiten und das Leben bei $[\ldots]^{14}$.

Gerade als eine Gebrauchsanweisung für Hinterwäldler, so soll man es wohl sehen, wird Moskau in beiden Filmen als unvergessliche Erfahrung postuliert, als eine Eingebung, die aus den Erinnerungen des jeweiligen Hauptstadtbesuchers nicht mehr zu tilgen ist. Dies betrifft gleichermaßen den aus Moskau stammenden, aber nicht mehr in Moskau wohnhaften Ingenieur Aljoša („И тот, кто в Москве побывает хоть раз, / не сможет ее позабыть“- „Wer in Moskau schon einmal gewesen ist, / wird es niemals vergessen können") und die in der Provinz gebore-

14 „Московское солнце повсюду горит, / повсюду лучи свои льет [...] / Москва окрыляет любого из нас / И учит работать и жить“. 
nen Figuren wie die Schweinepflegerin Glaša oder den Schafhirt Musaib („Друга я никогда не забуду, / если с ним подружился в Москве“ - „Ich werde den Freund (die Freundin) nie vergessen, / wenn ich ihn (sie) in Moskau kennengelernt habe").

$* * *$

Das Funktionieren der sowjetischen Hauptstadt der 1930er- und Anfang 1940er-Jahre als einer 'Verwandlungsmaschine', welche ohne den Zufluss der Provinzbewohner nicht leben und erst in der Peripherie ihr utopisches Potenzial entfalten kann, wirft ein paradoxes Licht auf die filmischen Projektionen der Moskauer Utopie. Dass ein weitestgehend 'provinzialisiertes' utopisches Zentrum die inländischen Fremden zu bereitwillig, wenn auch nur provisorisch, in seine Räume aufnimmt, mehr noch - dass es fast ausschließlich von Ortsfremden als Utopie gesehen werden kann und wird, unterscheidet es deutlich von den traditionellen Utopien bzw. Dystopien, die aus Sicherheitsgründen den fremden Blick und die Präsenz der Fremden konsequent vermeiden. Der Blick aus der Perspektive eines Moskauers, der sich als Großstadtbewohner definiert, ist unter diesen Bedingungen nur als verdeckter und unerwünschter möglich.

Dieser unerwünschte Blick ist in Das neue Moskau und in Die Schweinepflegerin unterschiedlich präsent. In Medvedkins Film, der die Repräsentanten der Hauptstadt noch zu Wort kommen lässt und sich gerade dadurch ideologisch angreifbar macht, ist der Blick 'von innen' wenigstens durch drei Figuren vertreten. Zunächst ist der Künstler Fedja zu nennen, der die Landschaften der schnell verschwindenden 'alten' Hauptstadt um die Wette malt, bevor sie buchstäblich weggeschleppt oder in die Luft gesprengt werden. Trotz seiner emphatischen Bemühungen, die Geschichte in piktoralen „Gedächtniskisten“ (Assmann 114-129) festzuhalten, hinkt er - und hinkt die durch ihn vertretene Malerei - einer von Ingenieuren und Bauarbeitern geformten Wirklichkeit hinterher. Fedjas Kapitulation vor den provinziellen Barbaren, die der Vernichtung historischer Gebäude Beifall schenken, wird im Film durch seine Lebensgeschichte bekräftigt. Denn indem er eine klassen- und regionenübergreifende Liaison mit Olja eingeht, wird er auch als Mann zum schülerhaften Kopierer Aljošas - genauso wie er früher als Maler die Realität kopieren musste, die der sibirische Ingenieur bereits überholt hatte $^{15}$. Zweitens ist der Moskauer Blick in Das neue Moskau durch die Audrey Hepburn-ähnliche Zoja vertreten. Gerade als Moskauerin lehnt sie, wie bereits erwähnt, den Vorschlag Aljošas, in die Taiga zu übersiedeln, mehrmals ab und ge-

${ }^{15}$ Zum Konflikt zwischen dem Ingenieur und dem Künstler im Film Medvedkins als Metapher einer Konkurrenz zweier Bildmedien, nämlich der Malerei und des Kinos, vgl. Drubek-Meyer 246-248. 
fährdet hierdurch ihre kommunistische Familienbildung. Erst viel später gibt sie, aufgeklärt durch das „lebendige Modell“", endlich nach, infiziert ihren fortschrittlichen Geliebten aber mit ihrem spießigen Ideal, so dass auch dieser in der Taiga von Piroggen und Quarkkuchen zu schwärmen beginnt. Drittens ist in diesem Zusammenhang die Figur der namenslosen Babuschka zu erwähnen, die sich am Ende des Films gegen Taiga und für Moskau entscheidet - trotz der ungemütlichen neuen Realität, die sie schon im früheren Handlungsverlauf mit surrealen Bildern der abgeschleppten Häuser konfrontierte. Es ist in diesem Sinne symptomatisch, dass es ausgerechnet die hochnäsige Zoja und die antiquierte Babuschka sind, die, ohne sich mit dem „lebendigen Modell“ auszukennen, dieses trotzdem einschalten müssen. Indem sie hierbei das Panorama des 'Fortschritts' rückwärts wiedergeben lassen und das bereits in der Utopie lebende Publikum zum Lachen bringen, beweisen sie ihre prinzipielle Rückständigkeit und Inkompatibilität mit der Zeit - genauso wie Fedja mit seinen rückwärtsgewandten Bildern.

In Die Schweinepflegerin, wo zwei kurze Moskauer Episoden als Präludien zur Übertragung des utopischen Impulses in die Provinz fungieren, werden dagegen alle expliziten Wahrnehmungen 'von innen' zur Freude der Parteikritiker konsequent ausgeklammert - als ein irrelevanter Faktor, der sich leicht ignorieren lässt. Die Verweise auf eine praktische, alltagstechnische Überlegenheit der Hauptstadt, die in Medvedkins Film bei der Wahl des Wohnorts eine wichtige Rolle spielen, tauchen in Die Schweinepflegerin nur einmal auf. Sie werden im Monolog des Schurken Kuz'ma angesprochen, der Glascha damit erfolglos anwirbt:

\footnotetext{
Von Schweinen haben wir auch zu Hause genug,

Moskau ist aber die Hauptstadt!

Hier herrschen die Großzügigkeit und die Weite, sie zu beschreiben reicht kein Papier aus.

Links ist der Promtorg, rechts der Mostorg

und verschiedene andere Kaufhäuser ${ }^{16}$.
}

Im übrigen Verlauf der Handlung ist die Moskauer točka zrenija (Uspenskij) nur in Andeutungen spürbar. Diese haben weniger mit der Bildsprache, vielmehr mit dem Wortlaut des Drehbuchs von dem Moskauer Autor Viktor Gusev zu tun. Es geht vor allem um einige ironisch gebrochene stilistische und semantische Verschiebungen - etwa dort, wo Glaša, von Kuz'mas Plädoyer für univermagi zuerst beeindruckt, über ein „marengofarbiges“ (cveta marengo) Kleid spricht oder wo sie und Musaib in Liedform versprechen, die „schönen Plätze, Gassen und

16 „Свиней-то и дома полно, / а Москва-то, гляди, столица! / Тут кругом широта и простор, / описать не хватит бумаги. / Налево - Промторг, направо - Мосторг / и разные прочие универмаги“. 
Brücken in Moskau" nie zu vergessen, obwohl sie diese gar nicht gesehen haben. Es sind diese Verschiebungen, die Pyrjevs singende Dörfler am Ende als stereotype Stilisierungen erscheinen lassen. Dem sozrealistischen Kanon angepasst, personifizieren sie die Repression des Urbanen, das in der „Kultur 1“ der 1920erJahre noch als Objekt eines begeisterten Kinodokumentarismus firmierte.

$* * *$

Die oben umrissene Spannung zwischen dem verordneten utopischen Diskurs, welcher die Differenz zwischen Stadt und Land aufhebt, und dem verdrängten Blick 'von innen', der einen solchen entdifferenzierten Raum als Pappenkulisse entlarvt, lässt die etwas weniger optimistische Kehrseite der Moskau-Inszenierungen der 1930er- und Anfang 1940er-Jahre erkennen. Denn trotz aller Unterschiede in Fokalisierungsstrategien und in den Artikulationen des als kleinbürgerlich abgestempelten Städtischen weisen sowohl das Scheitern der Stimmen des Ressentiments bei Medvedkin wie auch das Auslöschen nicht-ländlicher Perspektiven bei Pyrjev unmissverständlich auf die Frustration der Utopiebewohner hin - umso mehr, da die Hauptstädter, wie die Regisseure beider Filme, oft selbst aus der Provinz stammen ${ }^{17}$. Mit dieser Frustration reagieren die „verfaulten Intelligenzler“ (wie Fedja) und „kleinbürgerlichen“ Modedämchen aus der Hauptstadt (wie Zoja) auf die Invasion unermüdlicher Bauern und einfältiger provinzieller Visionäre. Diese schleichen sich in die kulturellen Räume des realen Moskaus ein und versuchen 'sogar', sich dort einzunisten, tilgen seine für sie unbedeutende Geschichte, stiften mit höchster Genehmigung ihre eigene Ordnung und kompensieren ihre eigenen Traumata - ohne auf die anderen zu achten, die mit diesen Einstellungen und Traumata nichts zu tun haben. Zugleich lässt sich darin die Befürchtung erkennen, die eigenen hauptstädtischen Privilegien einzubüßen und aus der Utopie herausgedrängt zu werden. Neben dem banalen „Verderben“ durch „die Wohnungsfrage“, von der Michail Bulgakovs Voland sprach (Bulgakow 195), vermittelt diese Befürchtung eine für die Atmosphäre der 1930er und Anfang 1940er Jahre durchaus typische Angst davor, dass ein solches Herausdrängen für den Verstoßenen auch ungefähr dort enden kann, wo der Regisseur Pyrjev geboren wurde und der Ingenieur Aljoša - vielleicht nicht ohne Absicht - die Idealstadt in Kleinkopie nachbaut. Die Frustration und die Angst bilden das verbotene und zumeist verschwiegene Unbewusste des Kinotextes über das Moskau der Vorkriegszeit. Dieses „cinematic unconscious“ fügt, wie Fabio Vighi mit Verweis auf Lacan ausführt, die Ordnungen des Symbolischen und des Realen zusammen (Vighi 17). Auf diese Weise lässt es den Zusammenhang zwischen der idealen Stadt und dem

${ }^{17}$ Medvedkin ist in Penza und Pyrjev im sibirischen Dorf Kamen' a Ob’ geboren und aufgewachsen. 
Kontext des stalinistischen Terrors wenigstens ansatzweise rekonstruieren, der in den Koordinaten des sozialistischen Realismus als äsopische Sprache und/oder als zum Teil ungewollte Sinnverschiebungen präsent ist.

\section{Literaturverzeichnis}

Assmann, Aleida. Erinnerungsräume. Formen und Wandlungen des kulturellen Gedächtnisses. München, Beck, 1999.

Augé, Marc. Nicht-Orte. Übersetzt von Michael Bischof. München, Beck, 2011.

Bohn, Thomas M. Minsk - Musterstadt des Sozialismus. Stadtplanung und Urbanisierung in der Sowjetunion nach 1945. Köln, Böhlau, 2008.

Brockmann, Stephen. A Critical History of German Film. Rochester (NY), Camden House, 2010.

Bulgakow, Michail. Der Meister und Margarita. Übersetzt von Ralf Schröder. Berlin, Volk \& Welt, 1995.

Bulgakowa, Oksana. „Symbolische Topographie des neuen Moskau im Film. Wie eine Stadt im Kopf entsteht“. Mastering Russian Spaces. Raum und Raumbewältigung als Probleme der russischen Geschichte. Hrsg. von Karl Schlögel unter Mitarbeit von Elisabeth Müller-Luckner. München, Oldenbourg, 2011, S. 253-278.

Chertenko, Alexander. „Možlyvo, mur buv metaforoju...“ Deščo pro istoriju odnieji fihury myslennja. Piznavaty kordony - perestupaty kordony - dolaty kordony. Hrsg. von Hans Richard Brittnacher, Ievgeniia Voloshchuk und Alexander Chertenko. Kyiv, Burago, 2011, S. 117-163.

Dobrenko, Evgenij. Politekonomija socrealizma. Moskau, Novoe literaturnoe obozrenie, 2007.

Dobrenko, Evgeniy. Stalinist Cinema and the Production of History: Museum of the Revolution. Edinburgh, Edinburgh University Press, 2008.

Drubek-Meyer, Natascha. „Primat des Doubles. Zwei sowjetische Filmkomödien: A. Medvedkins Novaja Moskva (1938) und G. Aleksandrovs Vesna (1947)“. Mystifikation, Autorschaft, Original. Hrsg. von Susi Frank, Renate Lachmann, Sylvia Sasse et al. Tübingen, Narr, 2001, S. 239-261.

Feuchtwanger, Lion. Moskau 1937. Ein Reisebericht für meine Freunde. Amsterdam, Querido, 1937. Groys, Boris. Die Erfindung Rußlands. München, Hanser, 1995.

Groys, Boris. Gesamtkunstwerk Stalin. Die gespaltene Kultur in der Sowjetunion. München, Hanser, 1988.

Hartmann, Anne. „Thomas Morus in Moskau. Die Sowjetunion der 1930er Jahre als Utopia“. Das Wort. Germanistisches Jahrbuch Russland, 2007, S. 103-117.

Jakovleva, Galina. „Ot universalij k unifikacii. Gradostroitel'nye koncepcii 1920-h godov i perioda 1930-h - načala 1950-h godov“. Architektura SSSR, 3, 1990, S. 28-36.

Lenin, V[ladimir]. Polnoe sobranie sočinenij, Bd. 44. Moskau, Izdatel'stvo političeskoj literatury, 1970.

Ljusyj, Aleksandr. Moskovskij tekst. Tekstologičeskaja koncepcija russkoj kul tury. Moskau, Veče, 2013.

Martin, Terry. The Affirmative Action Empire: Nations and Nationalism in the Soviet Union, 19231939. Ithaca-London, Cornell UP, 2001.

Neutatz, Dietmar. „«Schmiede der neuen Menschen» und Kostprobe des Sozialismus: Utopien des Moskauer Metrobaus“. Utopie und politische Herrschaft im Europe der Zwischenkriegszeit. Hrsg. von Wolfgang Hardtwig unter Mitarbeit von Philip Cassier. München, Oldenbourg, 2003, S. $41-56$. 
Papernyj, Vladimir. Kul'tura „dva“. Ann Arbor (MI), Ardis, 1985.

Revzin, Grigorij. „Moskva - fabrika utopij“. Kommersant, 49, 21.03.1998, S. 10. Web. 01.12.2020. https://www.kommersant.ru/doc/194958.

Ryklin, Michail. Räume des Jubels. Totalitarismus und Differenz: Essays. Übersetzt von Dirk Uffelmann. Frankfurt am Main, Suhrkamp, 2003.

Schlögel, Karl. „Utopie als Notstandsdenken - einige Überlegungen zur Diskussion über Utopie und Sowjetkommunismus“. Utopie und politische Herrschaft im Europe der Zwischenkriegszeit. Hrsg. von Wolfgang Hardtwig unter Mitarbeit von Philip Cassier. München, Oldenbourg, 2003, S. 77-96.

Schlögel, Karl. Terror und Traum. Moskau 1937. München, Hanser, 2008.

Shlapentokh, Dmitry, Vladimir Shlapentokh. Soviet Cinematography 1918-1991: Ideological Conflict and Social Reality. New York, De Gruyter, 1993.

Stites, Richard. Revolutionary Dreams: Utopian Vision and Experimental Life in the Russian Revolution. New York-Oxford, Oxford University Press, 1989.

Urussowa, Janina. Das neue Moskau. Die Stadt der Sowjets im Film 1917-1941. Köln, Böhlau, 2004.

Uspenskij, Boris. Poetik der Komposition. Struktur des künstlerischen Textes und Typologie der Kompositionsform. Übersetzt von Georg Mayer. Frankfurt am Main, Suhrkamp, 1975.

Vighi, Fabio. Traumatic Encounters in Italian Film: Locating the Cinematic Unconscious. Bristol (UK) Portland (OR), Intellect, 2006.

Voloshchuk, Ievgeniia. „Die «schöne neue Welt» des Sowjetimperiums in Joseph Roths Reportagenreihe «Reise in Rußland»“. Der lange Schatten des „Roten Oktober“. Zur Relevanz und Rezeption sowjet-russischer Kunst, Kultur und Literatur in Österreich 1918-1938. Hrsg. von Primus-Heinz Kucher und Rebecca Unterberger. Berlin, Lang, 2019, S. 133-148.

Widdis, Emma. Alexander Medvedkin. London-New York, I.B. Tauris, 2005. 